\title{
Semi-Targeted All-Pay Auctions: A Partial Exclusion Principle*
}

\author{
Matthias Dahm ${ }^{\dagger}$
}

September 7, 2018

\begin{abstract}
This paper studies the effects of a specific affirmative action policy in complete information all-pay auctions when players differ in ability. The contest organiser splits the overall prize of the competition into a targeted and an untargeted prize. The targeted prize is exclusively for disadvantaged (low-ability) agents and excludes advantaged agents partially from the overall prize. We consider a setting with one high-ability and two low-ability contestants and fully characterise equilibrium. Assuming that the contest organiser aims to maximise expected total effort, we show that (i) almost any targeted prize is preferable to a standard all-pay auction without targeted prize; (ii) the exclusion principle (Baye, Kovenock and de Vries, 1993) can be implemented by a wide range of sufficiently large targeted prizes; and (iii) partial exclusion by means of an appropriately chosen targeted prize benefits the organiser more than complete exclusion. We also discuss the robustness of our results in settings with more than three agents.

Keywords: Asymmetric contests, multi-prize contests, equality of opportunity, affirmative action, discrimination, prize structure, exclusion principle
\end{abstract}

JEL: C72, D72, J78

\section{Introduction}

I don't agree with opening up the Booker for the Americans, I think that's straightforwardly daft. The Americans have got enough prizes of their own.

*This paper supersedes the earlier version "All-Pay Auctions with Extra Prize: A Partial Exclusion Principle". I thank Luis Corchón for a conversation that prompted me to write this paper and three referees for useful comments. I am also grateful to José Alcalde, Luis Corchón, Gianni De Fraja, Patricia Esteve-González, Daniel Seidmann and participants at the CBESS Conference on 'Contests: Theory and Evidence 2016' in Norwich for valuable discussions and suggestions. The usual disclaimer applies.

${ }^{\dagger}$ University of Nottingham, School of Economics, University Park, Nottingham NG7 2RD, UK; ORCID: orcid.org/0000-0002-3146-6802; email: Matthias.Dahm@nottingham.ac.uk 
The idea of [the Booker] being Britain, Ireland, the old Commonwealth countries and new voices in English from around the world gave it a particular character and meant it could bring on writers. If you also include Americans - and get a couple of heavy hitters - then the unknown Canadian novelist hasn't got a chance.

\section{- Julian Barnes, Man Booker winner in 2011 1}

The above quote refers to a change in the rules for participation of the Man Booker Prize for Fiction. Initially, only novelists from the UK, Commonwealth, Ireland and Zimbabwe were eligible to receive the prize. With the new rules, all fiction in English published worldwide is eligible. This, of course, increases competition. Barnes is concerned that, because of the rule change, new novelists will lose against established writers and find it more difficult to win recognition. In this sense, we can think of the prize with the initial restricted participation rule as an affirmative action policy: The Booker prize is a targeted prize for some competitors, in addition to the main competition in which all novelists compete for recognition and book sales.2 The purpose of the present paper is to investigate the incentive effects of such a policy. We do so using an all-pay auction, which is a well-established tool for modeling competition. Our main result is that such a prize enhances competition. Consequently, even a contest designer who is not interested in affirmative action per se might decide to establish it.

The competition of novelists described above is a special case of a situation in which players compete by investing a costly and sunk resource in order to increase their probability of winning. In addition, some contestants have more options to receive a reward for their investment than others. This is not unusual $]^{3}$ Consider funding for research projects. All researchers in a given country might have access to funding from a central Government agency but only some regions might offer funding through a regional funding agency. $]^{4}$ Another example is Government funding for entrepreneurs. Young entrepreneurs

\footnotetext{
${ }^{1}$ See Mark Brown, 'Julian Barnes: letting US authors compete for Booker prize is 'daft' ', The Guardian, 27, November 2016.

${ }^{2}$ There is also another related affirmative action policy, the Woman's prize for fiction, "which was established in 1996 after the Booker failed to shortlist a single woman." See Alison Flood, 'Kamila Shamsie wins Women's prize for fiction for 'story of our times' ', The Guardian, 27, June 2018.

${ }^{3}$ Of course, there are also other international awards complemented by a prize for national competitors. For example, the City Council of Tarragona organises a fireworks competition that has an international prize and a prize for Catalan competitors. In 2009 both prizes were won by the same competitor. Another example is the category of Best Film. In 2011, the Catalan film 'Black Bread' won both the (Spanish) Goya Award and the (Catalan) Gaudí Award. Other film festivals establish additional prizes for example for youth, students or female contestants.

${ }^{4}$ Of course, besides affirmative action considerations, there could be other reasons to restrict competition to local competitors. At least in the Spanish research programme 'Proyectos Europa Excelencia' affirmative action considerations seem to be important, as participation requires that the proposal must have competed unsuccessfully for a 'Starting Grant' of the European Research Council.
} 
might have access to funding programmes that are only open to them, in addition to funding competitions that are open to all entrepreneurs.5 A last example is a prize for the best academic paper by a young scientist.

What distinguishes the examples above from the standard contest model of competition is that a player's prospects of receiving a reward for sunk investment depends not only on the magnitude of the investment but also on the player's identity. Players that belong to a disadvantaged group compete for a prize that others do not have access to. This creates a very specific prize structure. All agents compete for the untargeted prize, but only disadvantaged players contest the targeted prize. In this sense the competition is characterized by targeted rewards for sunk investments. This implies that even though there are two prizes, targeted prizes are different from second prizes. The reason is that second prizes, or consolation prizes, are not targeted. They can be won by all agents and change the prize structure for all agents in the same way. In contrast, targeted prizes reinforce the incentives of disadvantaged contestants to invest resources in the competition without affecting the prize structure for other agents. ${ }^{6}$

We investigate the effects of targeted prizes in an all-pay auction under complete information and with heterogeneous players. In our model agents differ in ability. The contest organiser splits the overall prize of the competition into an untargeted and a targeted prize. Only the low-ability agents contest the targeted prize. To discuss our contribution, it is useful to follow Baye et al. (1996) and to think of contestants with higher ability as stronger agents. Since in our model, however, a low-ability contestant can potentially win both the untargeted and the targeted prize, his behaviour depends not only on his ability but also on this split of the overall prize. To take this into account we modify Siegel's (2009) notion of the reach of a player to measure strength: a contestant's reach (or strength) is the highest effort level he can choose without obtaining a negative payoff if he wins all prizes that he contests with certainty. Our contribution is twofold.

Our first contribution is to fully characterise equilibrium in a setting with one highability and two low-ability contestants.7 We show that the introduction of the targeted prize weakens the high-ability agent and strengthens low-ability contestants. In the extreme, when the size of the targeted prize is large, the ranking of players in terms of

\footnotetext{
${ }^{5}$ Currently, the Spanish Ministry of Industry has such a programme for entrepreneurs younger than 40 years.

${ }^{6}$ Szymanski and Valletti (2005) investigate a contest between one strong and two weak contestants with a second prize. Assuming an imperfectly discriminating Tullock contest success function, rather than an all-pay auction, they show that with a second prize total effort might increase, provided the disadvantage of the weak agents is large enough.

${ }^{7}$ We discuss the contest with more than three agents and the robustness of our results in Section 5 . Our initial restriction to three contestants allows us to show uniqueness of equilibrium for almost all sizes of the targeted prize. This enables us to compare the contest with targeted prize unambiguously to a standard contest without targeted price, even though in the latter there is a multiplicity of equilibrium.
} 
their strengths is reversed. In our model, the targeted prize stimulates participation of disadvantaged contestants, because it will always be contested. Moreover, since disadvantaged contestants are symmetric, in equilibrium they use the same strategy. We show that the equilibrium is unique, unless the size of the targeted prize is equal to the relative difference in abilities and all agents are equally strong in terms of their reach. 8

Our second contribution is to provide a deeper understanding of the exclusion principle (Baye et al., 1993). This principle says that under some conditions the contest organiser benefits from excluding the most competitive contestants. The reason is that this can level the playing field and strengthen competition among the remaining agents. As a result total expected effort might increase. Our model allows us to relate the size of the targeted prize to the degree of exclusion of the advantaged player. Complete exclusion and no exclusion are extreme cases. Complete exclusion is obtained when the targeted prize is as large as possible and the untargeted prize is set to zero, while the contest without targeted prize results in no exclusion. Intermediate sizes of the targeted prize partially exclude the advantaged contestant from a part of the overall prize.

We show that complete exclusion is not necessary. Large targeted prizes weaken the advantaged contestant enough so that in equilibrium he abstains with certainty from the contest. This implies that partial exclusion via a targeted prize 'implements' complete exclusion as if finalists were selected directly 99 Moreover, we show that complete exclusion is not optimal. Choosing the size of the targeted prize roughly equal to the relative difference in abilities, the contest organiser can induce considerably higher expected total effort. Competition is strengthened, because this targeted prize levels the playing field completely (in terms of the reach of players). Since the advantaged contestant is only excluded from a part of the overall prize, a partial exclusion principle holds. Our analysis also implies that almost any targeted prize is preferable to a standard all-pay auction.10 Lastly, we provide an extension of our analysis to more than three contestants and highlight a novel finding. It might be beneficial for the contest organiser to design partial exclusion via a targeted prize in such a way as to discourage participation of an advan-

\footnotetext{
${ }^{8}$ When all contestants have the same reach there is a continuum of equilibria that arises from the possibility that the advantaged player might abstain with different probabilities from the contest. Equilibria with very related properties exist in the standard all-pay auction when agents have the same reach (Baye et al., 1996).

${ }^{9}$ Complete exclusion might not be feasible, for instance, for legal reasons.

${ }^{10}$ The reason for why we do not claim that any targeted prize is preferable to a standard all-pay auction is as follows. As the targeted prize goes to zero, the unique equilibrium becomes the equilibrium of the standard all-pay auction in which symmetric players use symmetric strategies. In the standard all-pay auction there is, however, another equilibrium in which only one of the symmetric players is active that has higher expected total effort. Thus depending on how agents coordinate in the standard all-pay auction, in equilibrium total expected effort might be higher in a standard all-pay auction than with a very small targeted prize.
} 
taged agent of intermediate ability but to provide sufficient incentives for an advantaged agent of high ability to participate. The targeted prize 'implements' a set of finalists in which an agent with intermediate ability is excluded but agents with high and low ability are included. This differs from the original exclusion principle in Baye et al. (1993) where the optimal set of finalists always includes pairs of adjacent contestants (in terms of ability) 11

Our results complement the analysis in Dahm and Esteve-González (2018). Dahm and Esteve-González investigate targeted prizes in a model with an imperfectly discriminating Tullock contest success function. In both models a targeted prize levels the playing field and increases the strength of competition. But, contrary to the present paper, Dahm and Esteve-González show that a targeted prize is only beneficial for intermediate levels of heterogeneity. In this sense the effect of a targeted prize is stronger when the contest success function is perfectly discriminatory. This helps to understand why the exclusion principle discovered by Baye et al. (1993) holds in the all-pay auction but, as shown by Fang (2002), does not hold in Tullock contests with economics of scale parameter equal to one 12

Since in our model there is an untargeted prize and a targeted prize, our paper contributes to the question under which conditions a contest organiser finds it optimal to establish more than one prize in all-pay auctions under complete information 13 Glazer and Hassin (1988) and Cohen and Sela (2008) provide conditions under which more than one prize should be established. Barut and Kovenock (1998), however, show that in their

\footnotetext{
${ }^{11}$ In Baye et al. (1993) contestants differ in their valuations for the prize. This corresponds to the abilities in our model.

${ }^{12}$ The exclusion principle does hold when the economics of scale parameter in a Tullock contest is large, so that the contest approaches the all-pay auction (Alcalde and Dahm, 2010). There are also other not perfectly discriminating contest success functions, different from Tullock's proportional form, for which the principle holds (Alcalde and Dahm, 2007). In recent work Matros and Rietzke (2017) investigate a model of contests on networks and provide conditions on the network under which the exclusion principle also applies to Tullock contests. A variation of the exclusion principle has also appeared in Konrad (2006). When firms have silent ownership shares in rivals, then a firm may be able to commit to abstain from the contest. This might be profitable, because it reduces the level of competition. Ownership shares, however, can also increase the level of competition, see Fu and Lu (2013). Menicucci (2006) and Bertoletti (2008) investigate the exclusion principle when the contest organiser is not fully informed about the contestants.

${ }^{13}$ The single-prize all-pay auction under complete information has been studied by Hillman and Samet (1987) and Hillman and Riley (1989), and its equilibrium has been completely characterised by Baye et al. (1996). An alternative to creating a targeted prize is to bias the selection rule in an all-pay auction. Such a setting has been analysed in Fu (2006), Li and Yu (2012), Pastine and Pastine (2012) and Franke et al. (2014 and 2018). Glazer and Hassin (1988), Barut and Kovenock (1998), Clark and Riis (1998) and Cohen and Sela (2008) analyse multiple-prize all-pay auctions under different assumptions concerning the contestants' valuations for prizes. Recent work by Siegel (2009, 2010 and 2014) and Xiao (2016) extends this model in many ways, including head starts. Sisak (2009) provides a review of the literature on multiple-prize contests.
} 
model the organiser is indifferent between establishing one or several prizes 14 Our model differs from these multiple-prize all-pay auctions, because in these papers prizes are not targeted to specific groups of contestants.

The paper is organised as follows. The next section presents our model of targeted prizes. We provide a full characterisation of equilibrium in Section 3 . Section 4 investigates expected total effort in equilibrium and establishes the partial exclusion principle. The extension to more than three contestants is provided in Section 5 and the last section contains concluding remarks. All proofs are relegated to an Appendix.

\section{Model}

There are three risk-neutral contestants with abilities $\alpha_{i}$. Agent 1 is advantaged and has high ability, while agents 2 and 3 are homogeneous and have low ability, so that $\alpha_{1} \geq \alpha_{d}>0$ with $d=2,3$. Our equilibrium characterisation in Section 3 allows for $\alpha_{1}=\alpha_{d}$ but for simplicity of the exposition in Section 4 we exclude the case in which all agents have the same ability. Contestants compete exerting effort $e_{i} \in \mathbb{R}_{+}$and different abilities are reflected in heterogeneous effort costs $c_{i}\left(e_{i}\right)=e_{i} / \alpha_{i}$. Effort is not recovered.

Contestants compete simultaneously for a budget $B$, which without loss of generality is normalized to one. The budget or overall prize is split into two prizes, the untargeted prize $(1-\beta)$ and the targeted prize $\beta$, with $\beta \in[0,1]$. Contestant 1 competes only for the untargeted prize $(1-\beta)$, while the disadvantaged contestants 2 and 3 compete for both prizes. In other words, the set of contestants $N=\{1,2,3\}$ compete for the untargeted prize (UP) of size $(1-\beta)$ and the set of agents $D=\{2,3\}$ contest the targeted prize (TP) of size $\beta$. Notice that this implies that, although contestants 2 and 3 exert effort only once, they might win both prizes. This structure of targeted rewards distinguishes our model from other contests with multiple prizes, including second prizes. The contest designer chooses $\beta$ in order to maximise total effort ${ }^{15}$

We consider an all-pay auction setting in which prizes are assigned as follows. Given a prize $k \in\{U P, T P\}$, let $A(k)$ be the set of agents competing for this prize. Then, given

\footnotetext{
${ }^{14}$ Moldovanu and Sela (2001) analyse a different all-pay auction model with incomplete information and show that when cost functions are convex several prices might be optimal; see also Liu and Lu (2017) for a related model. Clark and Riis (1998) investigate whether in a multiple-prize all-pay auction prizes should be awarded simultaneously or sequentially. Clark and Riis also show that the exclusion principle does not necessarily hold in multiple-prize all-pay auctions. Arbatskaya (2003), however, builds on the results by Barut and Kovenock (1998) and shows that a contest organiser might even in a symmetric setting benefit from excluding contestants, provided valuations depend on the number of contestants.

${ }^{15} \mathrm{~A}$ contest designer could also have different objectives, like competitive balance or the quality of the winner, see Serena (2017) for an insightful discussion. An interest in competitive balance or attaching a value to the participation by disadvantaged agents (perhaps on affirmative action grounds) provide different rationales for restricting competition than the logic highlighted in our analysis.
} 
a vector of efforts $e=\left(e_{1}, e_{2}, e_{3}\right)$, the win probability of agent $i \in A(k)$ for prize $k$ is

$$
p_{i}^{k}(e)=\left\{\begin{array}{ll}
\frac{1}{h} & \text { if } i \in A(k) \text { and } e_{i}=\max _{j \in A(k)}\left\{e_{j}\right\} \\
0 & \text { otherwise }
\end{array},\right.
$$

where $h$ is the number of agents in $A(k)$ exerting the highest effort (i.e., $h=\#\{j \in A(k)$ : $\left.\left.e_{j} \geq e_{j^{\prime}}, \forall j^{\prime} \in A(k)\right\}\right)$. Thus, player $i$ 's expected payoff is

$$
U_{i}(e)=(1-\beta) p_{i}^{U P}(e)+z_{i} \beta p_{i}^{T P}(e)-\frac{e_{i}}{\alpha_{i}},
$$

where $z_{i} \in\{0,1\}$ takes value 1 if $i \in D$, and value 0 otherwise. Note that this model includes two special cases. When $\beta=0$ or $\beta=1$ we obtain a standard all-pay auction without targeted prize in which the set of contestants is $N$ or $D$, respectively ${ }^{16}$

\section{Equilibrium characterization}

\subsection{Preliminaries}

It is useful to start by extending Siegel's (2009) notion of the "reach" to our model. In Siegel's model a contestant can win at most one prize and the reach of a contestant is the effort level at which the valuation for winning is zero. Since in our model a contestant can win more than one prize, we define contestant $i$ 's reach $r_{i}$ to be the effort level for which his valuation for winning all prizes that he contests is zero. More precisely,

$$
r_{i}=\max \left\{e_{i} \in \mathbb{R} \mid(1-\beta)+\beta z_{i}-\frac{e_{i}}{\alpha_{i}}=0\right\},
$$

with $z_{i} \in\{0,1\}$ taking value 1 if $i \in D$, and value 0 otherwise. Since rewards are targeted and the advantaged player does not compete for the targeted prize, (3) becomes

$$
r_{1}=\alpha_{1}(1-\beta) \text { and } r_{d}=\alpha_{d}
$$

for $d=2,3$. It follows that the order of contestants by their reach depends on $\beta$. We have that $r_{1} \geq r_{d}$ if and only if the size of the targeted prize is at most equal to the relative difference in abilities, that is,

$$
\beta \leq \frac{\alpha_{1}-\alpha_{d}}{\alpha_{1}} \equiv \hat{\beta}
$$

\footnotetext{
${ }^{16}$ In these situations the value of $\beta$ is such that there is only one prize. Consequently, the contestants eligible for this prize compete as in a standard all-pay auction. A similar situation arises if one disadvantaged agent does not contest the targeted prize. For instance, if agent 2 exerts zero effort with probability one, then players 1 and 3 compete (for the untargeted prize) as in a standard all-pay auction. The targeted prize is not contested and by exerting some positive effort contestant 3 receives it with certainty. We will also use the term standard all-pay auction to refer to such a situation.
} 
We will see that, as in other all-pay auctions, the second highest reach plays an important role in our analysis. In our simple three contestant model the second highest reach is $r_{d}$ for any size of the targeted prize $\beta{ }^{17}$

It is well known that in the standard all-pay auction without targeted prize there is no Nash equilibrium in pure strategies. This remains true when a targeted prize is introduced (formally this follows from Lemma 2 below). Consequently, we consider Nash equilibria in mixed-strategies. We represent the equilibrium mixed-strategy of contestant $i$ by the cumulative distribution function (cdf) $G_{i}\left(e_{i}\right)$. Agent $i$ randomises continuously on an interval $S$ if his mixed-strategy contains no mass points and has a strictly increasing cdf almost everywhere on $S$. We denote by $\gamma_{i}\left(e_{i}\right)$ the mass placed at $e_{i}$ by contestant $i$ 's mixed strategy. We say that a contestant $i$ is active if $\gamma_{i}(0)<1$. When $\gamma_{i}(0)=1$ we say that contestant $i$ abstains from the contest. Lastly, when $\gamma_{i}(0)=0$ we say that contestant $i$ never abstains from the contest.

We are now in a position to describe some basic properties of the equilibrium.

Lemma 1. For any $\beta>0$, in any equilibrium both disadvantaged contestants are active.

The previous lemma shows that a targeted prize is a powerful tool to make sure disadvantaged agents compete. The targeted prize will always be contested. The next lemma shows that disadvantaged contestants are not only active but never abstain. In addition, the next lemma establishes further important equilibrium properties.

Lemma 2. For any $\beta>0$, in any equilibrium the following holds:

1. Both disadvantaged contestants employ the same mixed-strategy $G_{2}=G_{3}$ and obtain an expected equilibrium payoff of zero.

2. For all $i$, there is no contestant $i$ who places mass on $\left(r_{d}, \infty\right), G_{i}$ contains no atoms in the half open interval $\left(0, r_{d}\right]$ and the disadvantaged contestants do not place an atom at zero.

As in other all-pay auctions, no contestant exerts more effort than the second highest reach $r_{d}$. Disadvantaged contestants use the same strategy, randomise continuously on $\left[0, r_{d}\right]$, place no atom anywhere, and dissipate all rents from the competition. The advantaged agent, however, might place an atom at zero. As in the standard all-pay auction without targeted prize, equilibrium implies that there are no atoms at points different

\footnotetext{
${ }^{17}$ Formally, the second highest reach is defined as $\hat{r} \in\left\{r_{1}, r_{2}, r_{3}\right\}$ such that the set $\left\{i \mid r_{i} \leq \hat{r}\right\}$ contains at least two agents and the set $\left\{i \mid r_{i}<\hat{r}\right\}$ contains at most one agent. Since $r_{2}=r_{3}=r_{d}$, we have that the second highest reach is $r_{d}$ for any size of the targeted prize $\beta$. In particular when $\beta>\hat{\beta}$, we have that $r_{d}>r_{1}$ but $\hat{r}=r_{d}$, as there are two disadvantaged contestants.
} 
from zero. Importantly, since Lemma 2 rules out that at equilibrium disadvantaged contestants use different strategies, it must hold that the set of active agents is either $D$ or $N$. It also allows us to use the notation $G_{1}\left(e_{1}\right)$ and $G_{d}\left(e_{d}\right)$ for the cdfs of the advantaged and disadvantaged agents' efforts $e_{1} \geq 0$ and $e_{d} \geq 0$, respectively, with $d=2,3$.

\section{2 $\quad$ Large targeted prizes}

It is reasonable to expect that the size of the targeted prize determines the characteristics of the equilibrium. On one hand, if the targeted prize is very small, one might expect it not to affect behaviour much, so that the equilibrium is similar to the standard three agent all-pay auction. On the other hand, if the targeted prize is very large, the advantaged contestant is excluded from a large part of the overall prize and one might expect the equilibrium to be similar to the standard two player all-pay auction among disadvantaged agents. In the present and the following subsection we show that this intuition is indeed true. We start with a large targeted prize.

Proposition 1. For any configuration of abilities $\alpha_{1} \geq \alpha_{d}$ the following holds:

(i) If and only if $\beta \geq \hat{\beta}$, there is a mixed-strategy equilibrium in which the advantaged agent abstains and disadvantaged agents play the same strategy characterized by the probability distribution function $G_{d}\left(e_{d}\right)$ for efforts $e_{d} \geq 0$ with

$$
G_{d}\left(e_{d}\right)=\left\{\begin{array}{ll}
\frac{e_{d}}{\alpha_{d}} & \text { if } e_{d} \in\left[0, r_{d}\right] \\
1 & \text { if } e_{d} \geq r_{d}
\end{array} .\right.
$$

The equilibrium payoff of all contestants is zero.

(ii) If $\beta>\hat{\beta}$, then the equilibrium described in part (i) is unique.

Notice that the previous proposition covers the case in which $\beta=1$, so that there is only the targeted prize and no untargeted prize. In this case the contest reduces to a standard all-pay auction in which only the disadvantaged agents compete, as the advantaged agent is completely excluded. Theorem 1 in Baye et al. (1996) states that in this case the unique equilibrium is as described in the statement 18 Proposition 1 shows that this unique equilibrium remains unchanged, provided the targeted prize is strictly larger than the relative difference in abilities. This implies that if there is not much difference in abilities even a small targeted prize can already be sufficient to discourage

\footnotetext{
${ }^{18}$ For completeness we mention that in Baye et al. (1996) contestants differ in their valuations for the prize, while in our model agents differ in ability. Starting with an equilibrium in Baye et al.'s model, it is, however, straightforward to modify the cdfs and obtain an equilibrium in the standard all-pay auction with heterogeneity in abilities.
} 
the advantaged agent from participating. The intuition for this is that, although in such a case the advantaged contestant is formally only excluded from a small part of the overall prize, this weakens him enough to convert the disadvantaged agents in the strong contestants (as measured by the reach of agents). As in Theorem 1 of Baye et al. (1996) a contestant exerts zero effort with probability one, if he competes against two strictly stronger players.

\subsection{Small targeted prizes}

We turn now to small targeted prizes. Since Lemma 2 implies that disadvantaged agents employ the same strategy, one might expect the equilibrium to be related to the symmetric equilibrium in the standard three agent all-pay auction. This intuition turns out to be correct. For convenience of the exposition we define the real number $r_{l}$ as the unique positive solution of

$$
\frac{r_{l}}{\alpha_{d}} \sqrt{\frac{\alpha_{1}}{(1-\beta)\left(\alpha_{1}(1-\beta)-\alpha_{d}+r_{l}\right)}}-\frac{\beta}{1-\beta}=0
$$

We are now in a position to state the following result.

Proposition 2. For any configuration of abilities $\alpha_{1} \geq \alpha_{d}$ the following holds:

(i) If and only if $\beta \leq \hat{\beta}$, there is a mixed-strategy equilibrium which is characterized by probability distribution functions $G_{1}\left(e_{1}\right)$ and $G_{d}\left(e_{d}\right)$ for the advantaged and disadvantaged agents' efforts $e_{1} \geq 0$ and $e_{d} \geq 0$, respectively, with

$$
G_{1}\left(e_{1}\right)= \begin{cases}0 & \text { if } e_{1} \in\left[0, r_{l}\right] \\ \frac{e_{1}}{\alpha_{d}} \sqrt{\frac{\alpha_{1}}{(1-\beta)\left(\alpha_{1}(1-\beta)-\alpha_{d}+e_{1}\right)}-\frac{\beta}{1-\beta}} & \text { if } e_{1} \in\left[r_{l}, r_{d}\right] \\ 1 & \text { if } e_{1} \geq r_{d}\end{cases}
$$

and

$$
G_{d}\left(e_{d}\right)=\left\{\begin{array}{ll}
\frac{e_{d}}{\alpha_{d}} & \text { if } e_{d} \in\left[0, r_{l}\right] \\
\sqrt{\frac{\alpha_{1}-\alpha_{d}}{\alpha_{1}(1-\beta)}+\frac{e_{d}}{\alpha_{1}(1-\beta)}-\frac{\beta}{1-\beta}} & \text { if } e_{d} \in\left[r_{l}, r_{d}\right] . \\
1 & \text { if } e_{d} \geq r_{d}
\end{array} .\right.
$$

The equilibrium payoff of the advantaged agent is $\hat{\beta}-\beta$, while the disadvantaged agents earn zero.

(ii) If $0<\beta<\hat{\beta}$, then the equilibrium described in part (i) is unique.

\footnotetext{
${ }^{19}$ In Appendix A.4 we show that for any $\alpha_{1}, \alpha_{d}$ and $\beta>0,77$ has a unique solution $r_{l}>0$.
} 
In the equilibrium described in the previous proposition, the advantaged contestant is aggressive, in the sense that he exerts a minimum effort of $r_{l}{ }^{20}$ This allows him to obtain a positive equilibrium payoff. Disadvantaged agents compete with the advantaged contestant for high effort levels and compete with each other for the targeted prize for low effort levels (lower than $r_{l}$ ). In fact, for low effort levels the disadvantaged agents compete as in (6) with the distributions rescaled by $1 / \beta$, the size of the targeted prize. As a result, the rent of disadvantaged agents is completely dissipated.

Notice that Proposition 2 covers the case in which $\beta=0$. In this case the contest reduces to a standard all-pay auction for the untargeted prize and there is no targeted prize. Baye et al. (1996) have shown that there is a continuum of equilibria, including one in which the two disadvantaged agents play the same strategy. Indeed, for $\beta=0$ we have that $r_{l}=0$ and (8) and (9) coincide with cdfs described in Theorem 2 in Baye et al. (1996) when the two disadvantaged agents play the same strategy. Proposition 2 indicates how this equilibrium changes as the targeted prize is introduced. In particular, it is straightforward to show that as $\beta$ increases, the disadvantaged contestants become more aggressive (in the sense of first-order stochastic dominance). In addition, when $\beta$ is small enough compared to $\hat{\beta}$, the advantaged contestant also seems to become more aggressive ${ }^{21}$ We will discuss these issues further in Section 4.

\subsection{Intermediate targeted prizes}

We consider now the special case of an intermediate targeted prize, which is equal to the relative difference in abilities. The analysis so far has already established that there exist at least two equilibria - one in which the advantaged contestant abstains $\left(\gamma_{1}(0)=1\right)$ and one in which he never abstains $\left(\gamma_{1}(0)=0\right)$. The next result shows that there is a continuum of equilibria and that the two aforementioned equilibria are extreme cases of this continuum. To state this result formally, it is convenient to introduce the following notation. Given a parameter $\gamma_{1}(0) \in[0,1]$, we define the real number

$$
\lambda \equiv\left((1-\beta) \gamma_{1}(0)+\beta\right)^{2} \alpha_{d}
$$

Notice that, since $\lambda$ is strictly increasing in $\gamma_{1}(0)$, we have that $\lambda \in\left[\beta^{2} \alpha_{d}, \alpha_{d}\right] \stackrel{22}{22}^{2}$

Proposition 3. Let $\beta=\hat{\beta}$. For any configuration of abilities $\alpha_{1}>\alpha_{d}$ there is a continuum of mixed-strategy equilibria in which disadvantaged agents play the same strategy

\footnotetext{
${ }^{20} \mathrm{~A}$ similar feature appears in a standard all-pay auction with additive bias in the function assigning the win probabilities, see $\mathrm{Li}$ and $\mathrm{Yu}(2012)$.

${ }^{21}$ When $\beta$ is close to $\hat{\beta}$, one can find configurations of abilities for which an increase in $\beta$ makes the advantaged contestant less aggressive.

${ }^{22}$ For simplicity we write $\lambda$ instead of $\lambda\left(\gamma_{1}(0)\right)$.
} 
and the advantaged contestant abstains with probability $\gamma_{1}(0) \in[0,1]$, where $\gamma_{1}(0)$ is a free parameter, and randomises continuously over the interval $\left[\lambda, r_{d}\right]$, where $G_{1}(\lambda)=\gamma_{1}(0)$. More precisely, the equilibrium is characterized by probability distribution functions $G_{1}\left(e_{1}\right)$ and $G_{d}\left(e_{d}\right)$ for the advantaged and disadvantaged agents' efforts $e_{1} \geq 0$ and $e_{d} \geq 0$, respectively, with

$$
G_{1}\left(e_{1}\right)= \begin{cases}\gamma_{1}(0) & \text { if } e_{1} \in[0, \lambda] \\ \frac{\alpha_{1}}{\alpha_{d}} \sqrt{\frac{e_{1}}{\alpha_{d}}}-\frac{\alpha_{1}-\alpha_{d}}{\alpha_{d}} & \text { if } e_{1} \in\left[\lambda, r_{d}\right] \\ 1 & \text { if } e_{1} \geq r_{d}\end{cases}
$$

and

$$
G_{d}\left(e_{d}\right)=\left\{\begin{array}{ll}
\frac{e_{d}}{\left((1-\beta) \gamma_{1}(0)+\beta\right) \alpha_{d}} & \text { if } e_{d} \in[0, \lambda] \\
\sqrt{\frac{e_{d}}{\alpha_{d}}} & \text { if } e_{d} \in\left[\lambda, r_{d}\right] . \\
1 & \text { if } e_{d} \geq r_{d}
\end{array} .\right.
$$

In any equilibrium the equilibrium payoffs of all contestants are zero.

The previous proposition bridges the equilibria in Propositions 1 and 2 . When $\gamma_{1}(0)=$ 1 , then $\lambda$ equals $r_{d}$ and (11) prescribes that the advantaged contestant abstains, while (12) becomes (6). As $\gamma_{1}(0)$ decreases, $\lambda$ decreases and the equilibrium has a similar structure to the one in Proposition 2 there is an interval of high effort levels on which all contestants are active and an interval of low effort levels on which only the disadvantaged agents are active contesting only the targeted prize. Again, for low effort levels the disadvantaged agents compete as in (6) with the distributions rescaled by $1 /\left((1-\beta) \gamma_{1}(0)+\beta\right)$, which represents the part of the overall prize that is uncontested by the advantaged agent. In the extreme, when $\gamma_{1}(0)=0$, then $\lambda$ is largest and Proposition 3 becomes the special case of Proposition 2 in which $\beta=\hat{\beta}$.

Moreover, since in Proposition 3 the size of the targeted prize $\hat{\beta}$ is equal to the relative difference in abilities, all contestants have the same reach. This has two implications that parallel the standard all-pay auction. First, all rents are completely dissipated and all equilibrium payoffs are zero. Second, the possibility that one contestant places mass at zero creates the possibility of equilibrium multiplicity. But while in Baye et al. (1996) the identity of the agent placing mass at zero is arbitrary, in our model the existence of the targeted prize implies that disadvantaged contestants never abstain from the contest ${ }^{23}$

\footnotetext{
${ }^{23}$ The case of three contestants with equal valuations (and therefore the same reach) is a special case of Theorem 1 in Baye et al. (1996). Proposition 3 covers this case by setting $\alpha_{1}=\alpha_{d}$, implying that the size of the targeted prize $\hat{\beta}$ is equal to zero. In this case 11 and 12 coincide with the mixed-strategies in Baye et al.'s symmetric three player example. Baye et al. have shown that there is a unique symmetric equilibrium and that there is a continuum of asymmetric equilibria in which one contestant places mass at 0 . The only difference between (11) and $(12)$ for $\alpha_{1}=\alpha_{d}$ and Baye et al. is that in our setting the identity of the contestant placing mass at 0 is not arbitrary.
} 


\section{A partial exclusion principle}

In this section we use the equilibrium characterizations of the previous section to compare partial exclusion of the advantaged agent by means of a targeted prize to two benchmarks. These benchmarks are, on one hand, a standard all-pay auction without targeted prize and, on the other hand, complete exclusion of the advantaged agent. As mentioned before, our model reduces to these benchmarks by setting $\beta=0$ and $\beta=1$, respectively. Since complete exclusion is only an interesting option for a contest organiser when abilities differ, we assume in this section that $\alpha_{1}>\alpha_{d}$.

Consider first the benchmark of $\beta=0$, which has been analysed in Baye et al. (1996). There is a continuum of equilibria that are not revenue equivalent. Revenue is maximized in the equilibria in which one disadvantaged agent abstains. For later reference we call this "best case" equilibrium the "asymmetric standard all-pay auction" equilibrium and state that it generates an expected sum of effort of $\alpha_{d}\left(\alpha_{d}+\alpha_{1}\right) /\left(2 \alpha_{1}\right)$. Revenue is minimized in the equilibrium in which the disadvantaged contestants use the same strategy. As we have seen the symmetric equilibrium is closely related to the equilibrium in Proposition 2

Consider now the case $\beta=1$, which also has been analysed in Baye et al. (1996). Complete exclusion of the advantaged contestant from the competition is a special case of our Proposition 1 and yields an expected sum of effort equal to $\alpha_{d}$. Our setting is a special case of Baye et al.'s (1993) exclusion principle. Consequently, our assumption $\alpha_{1}>\alpha_{d}$ implies that the organiser of the competition benefits from complete exclusion, as total expected effort in the "asymmetric standard all-pay auction" equilibrium is lower than $\alpha_{d}$. Since we know from Proposition 1 that the unique equilibrium under complete exclusion does not change for smaller but sufficiently large targeted prizes, we immediately have the following variation of the exclusion principle.

Proposition 4. Compared to a standard all-pay auction, the contest organiser benefits strictly from any targeted prize of size $\beta>\hat{\beta}$, because it implements the exclusion principle.

This result is interesting, because it implies that complete exclusion of the advantaged contestant is straightforward to implement. It is not necessary to formally exclude the advantaged agent from the competition, which might not be feasible for legal or ethical reasons. Such an explicit entry barrier can be avoided, because it suffices to establish a targeted prize that is larger than the relative difference in abilities. Interestingly, if this relative difference is small, say $1 / 6$, then the targeted prize can be quite small, for example $1 / 5$. 
Given Proposition 4, an important question is whether the contest organiser can do better than implementing the exclusion principle. Our main result reveals that this is the case.

Proposition 5. Compared to complete exclusion of the advantaged agent, the contest organiser benefits strictly from partial exclusion by means of a sufficiently large targeted prize of size $\beta<\hat{\beta}$.

The intuition for this result is simple. When the size of targeted prize is equal to the relative difference in abilities, there is a continuum of equilibria (Proposition 3). Broadly speaking these equilibria differ in the size of the atom that the advantaged contestant places at the origin. The smaller the size of the atom, the more aggressive the advantaged contestant becomes (in the sense of first-order stochastic dominance) and even though the disadvantaged agents become less aggressive, the first effect is stronger than the second. This implies that a targeted prize equal to the relative difference in abilities improves almost always strictly over complete exclusion. The only exception is the equilibrium in which the advantaged contestant plays a pure strategy and abstains from the contest, in which case the same result as under complete exclusion is obtained. ${ }^{24}$ Consider now a targeted prize of a size a little smaller than the relative difference in abilities. Because of the continuity of the cdfs and because for smaller targeted prizes the equilibrium is unique (Proposition 2), it follows that the contest organiser can be certain that partial exclusion by means of an appropriately chosen targeted prize improves strictly upon complete exclusion.

We summarise the discussion of this section with the help of Figure 1. The figure displays five examples. In each example we fix $\alpha_{1}=1$, while $\alpha_{d}$ takes values $1 / 10,1 / 4$, $1 / 2,3 / 4$ and $9 / 10$. To distinguish these cases we denote the threshold $\hat{\beta}$ by $\hat{\beta}^{\alpha_{d}}$. Given the values for $\alpha_{d}$, the thresholds $\hat{\beta}^{\alpha_{d}}$ are equal to $9 / 10,3 / 4,1 / 2,1 / 4$ and $1 / 10$. The horizontal axis indicates the size of the targeted prize $\beta$, while the vertical axis measures the expected sum of effort. Each curve represents a different value for $\alpha_{d}$ and includes for $\beta=0$ the equilibrium in which the disadvantaged contestants employ the same strategy. The isolated point higher but very close to each curve at $\beta=0$ indicates for each example total expected effort in the asymmetric standard all-pay auction equilibrium. In line with the exclusion principle, we see that $\beta=1$ generates strictly higher total expected effort than $\beta=0$. We also see that complete exclusion can be implemented by a wide range of sufficiently large targeted prizes. The vertical parts of the curves correspond to the continuum of equilibria when $\beta=\hat{\beta}^{\alpha_{d}}$. Choosing a targeted prize equal to the relative difference in abilities, the contest organiser cannot be worse-off than under complete

\footnotetext{
${ }^{24}$ In the proof of Proposition 5 in Appendix A.7 we provide expressions for individual and total expected effort as a function of the atom that the advantaged contestant places at the origin.
} 


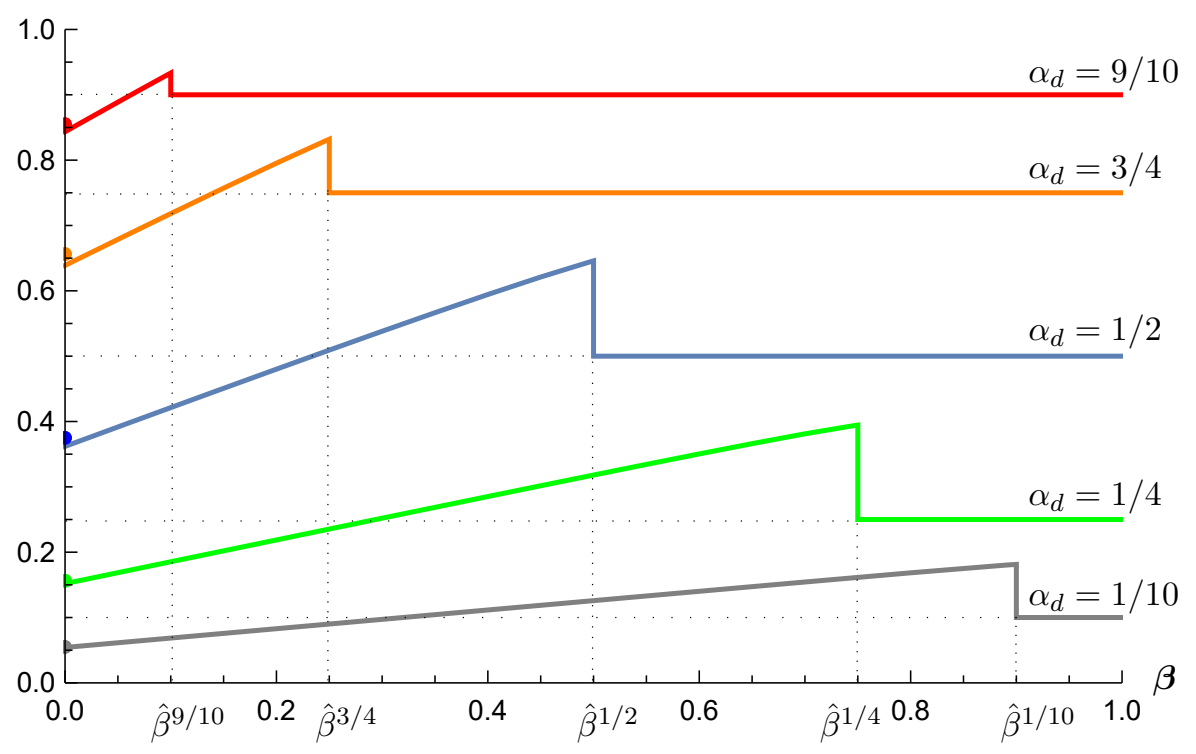

Figure 1: The size of the targeted prize and the sum of expected effort

exclusion. Total expected effort is maximized when the targeted prize is approximately equal to the relative difference in abilities 25 Notice also that a wide range of sizes for the targeted prize allow the contest organiser to strictly improve over complete exclusion and that almost any targeted prize improves over a standard all-pay auction.

\section{More than three agents}

In this section we discuss the robustness of our results when relaxing the assumption that there are only three agents. We start with the findings in Section 3 and show that in many instances the equilibria remain an equilibrium when there are more than three agents. In particular, this is true for the equilibrium in Proposition 3 that yields the highest sum of expected effort when the advantaged agent never abstains. We turn then to a discussion of the results of Section 4 and highlight a novel finding. It might be beneficial for the contest organiser to choose a targeted prize that 'implements' a set of finalists in which an agent with intermediate ability is excluded but agents with high and low ability are

\footnotetext{
${ }^{25}$ If the contest organiser is certain that the equilibrium is played in which the advantaged agent does not place mass at zero, then it is optimal to set the targeted prize equal to $\hat{\beta}$. If this is not the case, then he can avoid coordination on an unfavourable equilibrium by reducing the targeted prize a little bit. A technical issue concerns the existence of the optimal targeted prize. That may be solved by making the realistic assumption that a smallest monetary unit exists.
} 
included 26

We first investigate the robustness of the equilibria described in Propositions 13 when there are more than three agents. In order to do so we consider the original three-agent game and a modified game that arises by adding further agents to the original game. The next lemma relates the equilibrium of the original three-agent game to an equilibrium of the modified game. More precisely, it provides a condition under which the additional agents abstain from the contest and the original agents play the original equilibrium.

Lemma 3. Consider any $\beta>0$ and any equilibrium described in Propositions 1 3. Suppose the original game is modified by adding further agents. If

- for each additional disadvantaged agent, there exists a disadvantaged agent with higher ability in the original game and

- for each additional advantaged agent, there exists either a disadvantaged agent with higher ability in the original game or there exists an advantaged agent with higher ability receiving an equilibrium payoff of zero in the original equilibrium,

then it is an equilibrium in the modified game that the additional agents bid zero with probability one and the original agents bid as described in Propositions 13.

The intuition for this result is that the conditions in Lemma 3 identify for each additional agent an original agent receiving an equilibrium payoff of zero. The additional agent has, on one hand, higher effort cost than the original agent, as his ability is lower. On the other hand, for a given effort level, he also has a lower win probability in the modified game than the original agent in the original game, because the original agent has some chance of winning the modified game. Hence, it must be less profitable to exert this effort level than for the original agent and abstention is optimal.

Lemma 3 shows robustness of all the equilibria derived in Section 3 to the introduction of additional disadvantaged agents and for most of these equilibria to the introduction of additional advantaged agents. The only exception is the addition of advantaged agents with high ability in the context of a small targeted prize, when the original advantaged agent obtains positive equilibrium payoffs (Proposition 2). But it is clear that in such a situation the creation of a targeted prize is less interesting. To see this, consider a competition with two equally able advantaged agents. From Baye et al. (1993 and 1996) we know that (when $\alpha_{1}>\alpha_{d}$ ) in the unique equilibrium the expected sum of effort is $\alpha_{1}$, implying that this situation is already as competitive as possible and the exclusion principle does not hold. For other situations in which there is room to increase

\footnotetext{
${ }^{26}$ This differs from the original exclusion principle in Baye et al. (1993) where the optimal set of finalists always includes pairs of adjacent contestants (in terms of ability).
} 
the intensity of competition, however, Lemma 3 provides robustness. This includes the equilibrium in Proposition 3 that yields the highest sum of expected effort when the advantaged agent never abstains.

We turn now to the robustness of the results in Section 4. To fix ideas, suppose that there is a second advantaged agent with ability $\alpha_{4}$ such that $\alpha_{1}>\alpha_{4}>\alpha_{d}$. Notice first that Lemma 3 implies the robustness of Proposition 4. A sufficiently large targeted prize implements the exclusion principle ${ }^{27}$ Our main result in Proposition 5 is also robust in the sense that the equilibrium strategies in Proposition 3 remain part of an equilibrium in which the additional agents abstain. Introducing a targeted prize that provides partial exclusion, the contest organiser can never do worse than excluding the advantaged agents completely. But when in the competition with targeted prize the most advantaged agent is active, the organiser is strictly better off.

We conclude this section highlighting a novel result. In Baye et al. (1993) it is never beneficial for the contest organiser to choose a set of finalists that excludes an agent with an ability (that is, valuation in their model) between the abilities of the agents in the set of finalists. The reason is that the expected sum of effort is increasing in the second-highest ability. This is no longer true in the all-pay auction with targeted prize. Suppose again that there is a second advantaged agent with ability $\alpha_{4}$ such that $\alpha_{1}>\alpha_{4}>\alpha_{d}$ but let $\alpha_{4}$ be close to $\alpha_{d}$. When there is no targeted prize, we know from Baye et al. (1996) that there is a unique equilibrium in which the two disadvantaged agents abstain. Since $\alpha_{4}$ is close to $\alpha_{d}$, we know from Baye et al. (1993, p. 293) that the contest organiser benefits from complete exclusion of both advantaged agents. Setting a targeted prize equal to the relative difference in abilities $\beta=\hat{\beta}$, however, weakens the advantaged agent with lower ability sufficiently to induce him to abstain. As discussed before, with the exception of the equilibrium in which the most advantaged agent bids zero with probability one, the organiser is strictly better-off than with complete exclusion of both advantaged agents. Thus, it is beneficial for the contest organiser to use the targeted prize to exclude only the agent with the second-highest ability.

\section{Concluding remarks}

This paper analyses the effects of establishing a targeted prize for disadvantaged agents in an all-pay auction under complete information. The overall prize value is split in a targeted

\footnotetext{
${ }^{27}$ The contest organiser benefits from complete exclusion, provided $\alpha_{4}$ is low enough. The precise condition $\alpha_{4}\left(\alpha_{4}+\alpha_{1}\right) /\left(2 \alpha_{1}\right)<\alpha_{d}$ is the same as in Baye et al. (1993, p. 293). While a rigorous proof of uniqueness of equilibrium is beyond the scope of this section, it should be noted that here and in the following discussion there are at least two agents with strictly higher reach than agent 4 and that agent 4 competes only for the untargeted prize. In any equilibrium of the standard single-prize all-pay auction such an agent bids zero with probability one, see Baye et al. (1996).
} 
prize and an untargeted prize. All types of contestants compete for the untargeted prize, but only low-ability agents can win the targeted prize. We fully characterise equilibrium in a setting with one high-ability and two low-ability contestants. Assuming that the contest organiser aims to maximize expected total effort, our main result establishes a partial exclusion principle. Partial exclusion of the advantaged agent from part of the overall prize value by means of an appropriately chosen targeted prize benefits the organiser more than complete exclusion.

We also show that almost any targeted prize is preferable to a standard all-pay auction. This result is robust to a variation of our informational assumptions. Following Menicucci (2006) and Bertoletti (2008) assume that the organiser knows less about abilities than the agents. More precisely, suppose that the contest organiser can distinguish between advantaged and disadvantaged contestants but does not know the exact abilities. In addition, assume that contestants still know each others' abilities. In such a situation, given a size of the targeted prize, the equilibria we have characterised will remain unchanged. Without further information, however, the organiser will not be able to choose the size of the targeted prize that maximises total expected effort. But as we have seen he will still be very likely to be able to improve upon a standard all-pay auction. And if the organiser has some idea about relative abilities, then he might even do considerably better than with complete exclusion.

An interesting avenue for future research is to generalise the prize structure of our model of targeted prizes to a more general model of targeted rewards for sunk investments. For instance, our model is a special case of a multiple-prize all-pay auction in which the set of agents competing for each of the prizes is (potentially) different. Such a model might capture interesting features of real contests and might allow to ask novel questions regarding contest design. Concerning design, one could ask which agents should compete for which prizes in order, say, to maximise total effort. Concerning the task to build more realistic contest models, consider lobbying. Interest groups are usually affected by many different policies, but not all groups care about all issues. Our analysis of targeted prizes suggests that interest groups affected by multiple issues compete harder. Lastly, consider the contests for funding of research projects mentioned in the Introduction ${ }^{28}$ Say there are two regions. Each of the regions offers a funding competition, in addition to the competition organised by the central Government agency. What is the optimal degree of decentralization of research funds?

\footnotetext{
${ }^{28}$ Beviá and Corchón (2015) compare centralized and decentralized contests for example for research funds but do not allow that a contestant competes at the same time in both contests.
} 


\section{A Appendix}

In this Appendix we provide a proof for the results stated in the main text. We use the notation $u_{i}\left(e_{i}, G_{-i}\right)$ to indicate contestant $i$ 's payoff from bidding $e_{i}$ when the other two agents employ strategies $G_{-i}$. We indicate by $\underline{s}_{i}$ and $\bar{s}_{i}$ the lower and upper bounds of the supports of player $i$ 's mixed-strategy, respectively. Contestant $i$ 's expected equilibrium payoff is indicated by $u_{i}^{*}$.

\section{A.1 Proof of Lemma 1}

Without loss of generality suppose that contestant 2 abstains. In such a case agent 3 receives the targeted prize and competes with contestant 1 in a standard all-pay auction for the untargeted prize of size $(1-\beta)$. From Baye et al. (1996) we know that in the unique equilibrium of this all-pay auction the upper bound of the mixed-strategies of contestants 1 and 3 is $(1-\beta) \alpha_{3}$. Suppose now contestant 2 deviates and bids $\hat{e}=(1-\beta) \alpha_{3}$. This yields a higher expected payoff than abstaining, as $u_{2}\left(\hat{e}, G_{-2}\right)=1-(1-\beta) \alpha_{3} / \alpha_{2}=\beta>0$, since the disadvantaged contestants 2 and 3 have the same ability.

Q.E.D.

\section{A.2 Proof of Lemma 2}

We prove Lemma 2 through a series of claims.

Claim 1. $\bar{s}_{i} \leq r_{d}$ for all $i$.

Proof: Notice that there is no contestant $i$ who employs a strategy that places mass on $\left(r_{i}, \infty\right)$. The reason is that it implies strictly negative payoffs, while setting $e_{i}=0$ avoids losses. Thus, we have $\bar{s}_{i} \leq r_{i}$ for all $i$. Suppose $\beta \geq \hat{\beta}$. In this case we have that $r_{1} \leq r_{d}$, implying the statement. Suppose now $\beta<\hat{\beta}$, in which case we have that $r_{1}>r_{d}$. But since disadvantaged agents do not put mass on $\left(r_{d}, r_{1}\right]$, contestant 1 has no incentive to use a strategy with $\bar{s}_{1}>r_{d}$.

Q.E.D.

Claim 2. $\underline{s}_{d}=0, \gamma_{d}(0)=0$ and $u_{d}^{*}=0$, for $d=2,3$.

Proof: Suppose the first statement is not true and without loss of generality let $\underline{s}_{2} \geq \underline{s}_{3}$ with $\underline{s}_{2}>0$. There are three cases to consider.

1. $\underline{s}_{2}=\underline{s}_{3}>0$. On one hand, if there does not exist $d$ with $d=2,3$ such that $\gamma_{d}\left(\underline{s}_{d}\right)>0$, then $u_{2}\left(\underline{s}_{2}, G_{-2}\right)=-\underline{s}_{2} / \alpha_{2}<0$, as (irrespective of the advantaged player 1's effort) contestant 2 cannot win any of the prizes with positive probability and does not recover his effort. On the other hand, if such a $d$ exists, say $d=2$, then the other disadvantaged contestant 3 could profitably increase $\underline{s}_{3}$ slightly, as this strictly increases at least the probability of winning the targeted prize and the costs of the additional effort can be made arbitrarily small. 
2. $\underline{s}_{2}>\underline{s}_{3}>0$. Similar to the previous case, we have that $u_{3}\left(\underline{s}_{3}, G_{-3}\right)=-\underline{s}_{3} / \alpha_{3}<0$ and contestant 3 could profitably reduce $\underline{s}_{3}$ to zero.

3. $\underline{s}_{2}>\underline{s}_{3}=0$. There are again three cases.

(a) $\underline{s}_{2}=\underline{s}_{1}$. On one hand, if $\gamma_{2}\left(\underline{s}_{2}\right)=0$, then $u_{1}\left(\underline{s}_{1}, G_{-1}\right)=-\underline{s}_{1} / \alpha_{1}<0$, as contestant 1 cannot win the untargeted prize and expends effort. On the other, if $\gamma_{2}\left(\underline{s}_{2}\right)>0$, then by analogous reasoning to the first case contestant 1 could profitably increase $\underline{s}_{1}$ slightly.

(b) $\underline{s}_{2}>\underline{s}_{1}$. It follows that $\underline{s}_{1}$ must be equal to zero, because otherwise $u_{1}\left(\underline{s}_{1}, G_{-1}\right)=$ $-\underline{s}_{1} / \alpha_{1}<0$. Similarly, for any $i=1,3$ bidding $e_{i}$ such that $\underline{s}_{2}>e_{i}>0$ is unprofitable and bidding $e_{i}=\underline{s}_{2}$ can only be profitable if $\gamma_{2}\left(\underline{s}_{2}\right)>0$. In the latter case, however, contestant $i$ could, as in cases 1 and 3(a), profitably increase $e_{i}$ slightly. Thus $G_{i}\left(\underline{s}_{2}\right)=G_{i}(0)$ must hold for $i=1,3$. This implies that contestant 2 could profitably decrease $\underline{s}_{2}$.

(c) $\underline{s}_{2}<\underline{s}_{1}$. Again, any bid $e_{3}$ with $\underline{s}_{2}>e_{3}>0$ is unprofitable and $e_{3}$ with $e_{3}=\underline{s}_{2}$ can only be profitable if $\gamma_{2}\left(\underline{s}_{2}\right)>0$. In the latter case, however, contestant 3 could (similarly to case 1 ) profitably increase $e_{3}$ slightly. Thus $G_{3}\left(\underline{s}_{2}\right)=G_{3}(0)$ must hold, in which case contestant 2 could profitably decrease $\underline{s}_{2}$.

This proves that $\underline{s}_{d}=0$.

Consider now the second statement. Without loss of generality let $\gamma_{2}(0)>0$. Contestant 3 has an incentive to raise $\underline{s}_{3}$ by $\epsilon>0$ but very small.

Lastly, consider the third statement. Without loss of generality consider contestant 2 and notice that $\gamma_{3}(0)=0$ implies $u_{2}^{*}=u_{2}\left(0, G_{-2}\right)=0$.

Q.E.D.

Claim 3. For all $i, G_{i}$ contains no atoms in the half open interval $\left(0, r_{d}\right]$.

Proof: Suppose the cdf of one of the disadvantaged agents, say $G_{2}$, has an atom at $\tilde{e}_{2} \in\left(0, \alpha_{d}\right]$. Suppose $\bar{s}_{1} \geq \tilde{e}_{2}$. In such a case contestant 3's win probability for the targeted prize of size $\beta$ has an upward jump at $\tilde{e}_{2}$. Suppose $\bar{s}_{1}<\tilde{e}_{2}$. In such a case contestant 1's win probability for the untargeted prize and contestant's 3 win probability for both prizes have an upward jump at $\tilde{e}_{2}$. In both cases adapting the argument in the proof of Lemma 5 in Baye et al. (1996) allows to conclude that there must be an $\epsilon$-neighbourhood below $\tilde{e}_{2}$ in which neither contestant 1 nor 3 put mass, implying that it is not an equilibrium strategy for agent 2 to put mass at $\tilde{e}_{2}$. Suppose now $G_{1}$ has an atom at $\tilde{e}_{1} \in\left(0, \alpha_{d}\right]$. The fact that the win probability of contestants 2 and 3 for the untargeted prize has an upward jump at $\tilde{e}_{1}$ allows to reach a similar contradiction.Q.E.D.

Claim 4. Suppose $e \in\left(0, r_{d}\right]$ is a point of increase in $G_{i}$ for $i \in D$. Then $e$ is also a point of increase in $G_{j}$ for all $j \in D$. 
Proof: We start with an observation. Consider the disadvantaged contestants and denote them $i$ and $j$. If $e \in\left(0, \alpha_{d}\right]$ is a point of increase in $G_{i}$, then contestant $i$ must receive his equilibrium payoff at $e 2^{29}$ Using claim 2 we see that

$$
u_{i}\left(e, G_{-i}\right)=G_{j}(e)\left(G_{1}(e)(1-\beta)+\beta\right)-\frac{e}{\alpha_{d}}=0 .
$$

Since $e$ might or might not be a point of increase in $G_{j}$ it follows from claim 2 that

$$
u_{j}\left(e, G_{-j}\right)=G_{i}(e)\left(G_{1}(e)(1-\beta)+\beta\right)-\frac{e}{\alpha_{d}} \leq 0 .
$$

Expressions (13) and (14) imply that

$$
\left(G_{i}(e)-G_{j}(e)\right)\left(G_{1}(e)(1-\beta)+\beta\right) \leq 0 .
$$

Since $G_{1}(e)(1-\beta)+\beta>0$, we conclude that

$$
G_{i}(e) \leq G_{j}(e)
$$

Without loss of generality suppose that $y \in\left(0, \alpha_{d}\right]$ is a point of increase in $G_{2}$ but not in $G_{3}$. Since by Claims 2 and 3 there are no mass points in $\left[0, \alpha_{d}\right]$ and using the definition of a cdf, there must exist $z \neq y$ with $z \in\left(0, \alpha_{d}\right]$ such that $G_{3}(z)=G_{2}(y)$ and $z$ is a point of increase in $G_{3}$. There are two cases to consider.

1. $z<y$. Since $z$ is a point of increase in $G_{3}$, we can apply $(16)$ and establish that $G_{3}(z) \leq G_{2}(z)$. By the properties of a cdf we have that $G_{2}(z) \leq G_{2}(y)$. Thus we obtain $G_{3}(z) \leq G_{2}(z) \leq G_{2}(y)$. Our initial assumption that $G_{3}(z)=G_{2}(y)$ allows to establish that

$$
G_{3}(z)=G_{2}(z)=G_{2}(y)
$$

must hold. Therefore each $w \in(z, y)$ cannot be a point of increase in $G_{2}$ and, since each point must be a point of increase for at least two contestants (Lemma 7 in Baye et al., 1996, applies), $w$ must be a point of increase in $G_{3}$. Applying (16) allows then to establish that $G_{3}(w) \leq G_{2}(w)$. By the properties of a cdf we obtain $G_{3}(z) \leq G_{3}(w) \leq G_{2}(w) \leq G_{2}(y)$. But from 17 we see that for each $w \in(z, y)$ these weak inequalities must hold with strict equality, contradicting that $z$ is a point of increase in $G_{3}$.

\footnotetext{
${ }^{29}$ We use the following definition of a point of increase. Consider a function $f: A \rightarrow \mathbb{R}$, defined on a convex set $A \subset \mathbb{R}$. The point $e_{0}$ is a point of increase in $f$ if for all $\epsilon>0$, there exists $e \in\left(e_{0}, e_{0}+\epsilon\right)$ such that $f(e)>f\left(e_{0}\right)$.
} 
2. $z>y$. Since $y$ is a point of increase in $G_{2}$, we can apply $(16)$ and establish that $G_{2}(y) \leq G_{3}(y)$. By the properties of a cdf we have that $G_{3}(y) \leq G_{3}(z)$. Thus we obtain $G_{2}(y) \leq G_{3}(y) \leq G_{3}(z)$. Our initial assumption that $G_{3}(z)=G_{2}(y)$ allows to establish that

$$
G_{2}(y)=G_{3}(y)=G_{3}(z)
$$

must hold. Therefore each $w \in(y, z)$ cannot be a point of increase in $G_{3}$ and $w$ must be a point of increase in $G_{2}$. Applying (16) allows then to establish that $G_{2}(w) \leq$ $G_{3}(w)$. By the properties of a cdf we obtain $G_{2}(y) \leq G_{2}(w) \leq G_{3}(w) \leq G_{3}(z)$. But from (18) we see that for each $w \in(y, z)$ these weak inequalities must hold with strict equality, contradicting that $y$ is a point of increase in $G_{2}$.

Q.E.D.

Claim 5. Suppose $e \in\left(0, r_{d}\right]$ is a point of increase in $G_{2}$ and $G_{3}$. Then $G_{2}=G_{3}$ at e.

Proof: Claim 2 implies that (13) and (14) must both hold with equality. Thus (16) must hold with equality too.

Q.E.D.

Claim 6. $\bar{s}_{d}=r_{d}$, for $d=2,3$.

Proof: Claims 4 and 5 imply $\bar{s}_{2}=\bar{s}_{3}$. Let $\bar{s}_{2}=\bar{s}_{3}<\alpha_{d}$. Since $\bar{s}_{2}=\bar{s}_{3}$ holds, contestant 1 has no incentive to use a strategy with $\bar{s}_{1}>\bar{s}_{2}$. Consider the payoff to contestant 2 from bidding $\bar{s}_{2}$. This yields $u_{2}\left(\bar{s}_{2}, G_{-2}\right)=1-\bar{s}_{2} / \alpha_{d}>0$, contradicting Claim 2. Q.E.D.

Claim 7. $G_{2}(e)=G_{3}(e)$, for all $e \in\left[0, r_{d}\right]$.

Proof: Since each point $e \in\left(0, r_{d}\right]$ must be a point of increase for at least two contestants (Lemma 7 in Baye et al., 1996, applies) and using Claim 4, we conclude that each point $e \in\left(0, r_{d}\right]$ is a point of increase for contestants 2 and 3. Applying Claim 5 we have that $G_{2}(e)=G_{3}(e)$, for all $e \in\left(0, r_{d}\right]$, implying that $G_{2}(e)=G_{3}(e)$ must hold at $e=0$.Q.E.D.

Lemma 2 follows directly from Claims 17.

Q.E.D.

\section{A.3 Proof of Proposition 1}

We start with part (i). Under the assumption that disadvantaged agents employ (6), the expected payoff of the advantaged contestant from any $e_{1}$ is

$$
u_{1}\left(e_{1}, G_{-1}\right)=\left\{\begin{array}{ll}
\left(\frac{e_{1}}{\alpha_{d}}\right)^{2}(1-\beta)-\frac{e_{1}}{\alpha_{1}} & \text { if } e_{1} \in\left[0, \alpha_{d}\right] \\
(1-\beta)-\frac{e_{1}}{\alpha_{1}} & \text { if } e_{1} \geq \alpha_{d}
\end{array} .\right.
$$


Suppose $\beta \geq \hat{\beta}$ and that disadvantaged agents employ (6). Since $\beta \geq \hat{\beta}$ is equivalent to $r_{d} \geq r_{1}$ and since agent 1 only considers deviations to $e_{1} \in\left[0, r_{1}\right]$, we have that $\alpha_{d} \geq(1-\beta) \alpha_{1} \geq e_{1}$ must hold. Hence for each such $e_{1}$ we have that $U_{1}\left(e_{1}\right) \leq 0$ if and only if

$$
e_{1} \leq \frac{\left(\alpha_{d}\right)^{2}}{\alpha_{1}(1-\beta)}
$$

which under our assumptions is true. Lastly, observe that when the advantaged agent exerts zero effort with probability one, the disadvantaged contestants compete as in a standard two player all-pay auction. From Hillman and Riley (1989) and Baye et al. (1996), we know that in such a situation the (unique) equilibrium is characterized by (6). Thus, the strategies in the statement constitute an equilibrium, because for each agent, given the strategies of the other players, there is no positive effort level that yields a strictly higher payoff.

Now let $\beta<\hat{\beta}$. Suppose that the advantaged agent 1 abstains and that disadvantaged agents employ (6). Agent 1 can profitably deviate to $e_{1}=\alpha_{d}$, as

$$
u_{1}\left(e_{1}, G_{-1}\right)=(1-\beta)-\frac{\alpha_{d}}{\alpha_{1}}>0 \Leftrightarrow \alpha_{d}<(1-\beta) \alpha_{1},
$$

which is equivalent to $\beta<\hat{\beta}$.

Consider now part (ii). By Lemma 2 the set of active agents is either $D$ or $N$. If it is $D$, then, as already mentioned, the unique equilibrium is described in part (i). So suppose the set of active agents is $N$. In such a case there must exist $e \in\left(0, r_{d}\right]$ which is a point of increase for all three contestants. In the proof of Proposition 2 below we show that the only compatible cdfs are described in (8) and (9) and that this implies that $u_{1}\left(e, G_{-1}\right)=\hat{\beta}-\beta<0$. Under the assumption that $\beta>\hat{\beta}$, contestant 1 is strictly better-off abstaining and thus the equilibrium is unique.

Q.E.D.

\section{A.4 Equation (7) has a unique solution}

Notice that the left hand side of (7) is continuous. Moreover, since $\beta>0$, (7) is strictly negative at $e_{1}=0$ and equal to one at $e_{1}=\alpha_{d}$. Applying Bolzano's Theorem we conclude that there exists $r_{l}$ such that (7) holds. It can be shown that the left hand side of (7) is strictly increasing in $r_{l}$. Thus there is a unique $r_{l}$ such that (7) holds.

Q.E.D.

\section{A.5 Proof of Proposition 2}

Notice first that $G_{1}\left(e_{1}\right)$ and $G_{d}\left(e_{d}\right)$ as defined in (8) and (9) are well defined distribution functions. In particular, since the function in the second branch of $(8)$ is the left hand side of (7), we have already established that its density function is strictly positive. It 
can also be shown that the first and the second branch of (9) intersect for the effort level $r_{l}{ }^{30}$

Consider now part (i). Suppose $\beta \leq \hat{\beta}$ and that contestant 1 and one disadvantaged agent, say contestant 2 , employ (8) and (9). The expected payoff of contestant 3 for any $e_{3}$ is then

$$
u_{3}\left(e_{3}, G_{-3}\right)=\left\{\begin{array}{ll}
\frac{e_{3}}{\alpha_{d} \beta} \beta-\frac{e_{3}}{\alpha_{d}} & \text { if } e_{3} \in\left[0, r_{l}\right] \\
\sqrt{\frac{\alpha_{1}-\alpha_{d}}{\alpha_{1}(1-\beta)}+\frac{e_{3}}{\alpha_{1}(1-\beta)}-\frac{\beta}{1-\beta}} \frac{e_{3}}{\alpha_{d}} \sqrt{\frac{\alpha_{1}(1-\beta)}{\alpha_{1}(1-\beta)-\alpha_{d}+e_{3}}}-\frac{e_{3}}{\alpha_{d}} & \text { if } e_{3} \in\left[r_{l}, \alpha_{d}\right] . \\
1-\frac{e_{3}}{\alpha_{d}} & \text { if } e_{3} \geq \alpha_{d}
\end{array} .\right.
$$

Simplifying allows to conclude that for any $e_{3} \geq 0$ we have $u_{3}\left(e_{3}, G_{-3}\right) \leq 0$, with strict equality for $e_{3} \in\left[0, \alpha_{d}\right]$.

Suppose now that agents 2 and 3 follow the strategies in the statement. Consider agent 1 . We have that

$$
u_{1}\left(e_{1}, G_{-1}\right)=\left\{\begin{array}{ll}
\left(\frac{e_{1}}{\alpha_{d} \beta}\right)^{2}(1-\beta)-\frac{e_{1}}{\alpha_{1}} & \text { if } e_{1} \in\left[0, r_{l}\right] \\
\left.\frac{\alpha_{1}-\alpha_{d}}{\alpha_{1}(1-\beta)}+\frac{e_{1}}{\alpha_{1}(1-\beta)}-\frac{\beta}{1-\beta}\right)(1-\beta)-\frac{e_{1}}{\alpha_{1}} & \text { if } e_{1} \in\left[r_{l}, \alpha_{d}\right] . \\
1-\beta-\frac{e_{1}}{\alpha_{1}} & \text { if } e_{1} \geq \alpha_{d}
\end{array} .\right.
$$

For $e_{1} \in\left[r_{l}, \alpha_{d}\right]$ we obtain $u_{1}\left(e_{1}, G_{-1}\right)=\hat{\beta}-\beta$, while $u_{1}\left(e_{1}, G_{-1}\right)$ decreases strictly with $e_{1}$ for $e_{1}>\alpha_{d}$. Consider $u_{1}\left(e_{1}, G_{-1}\right)$ for $e_{1} \in\left[0, r_{l}\right]$. It is straightforward to show that $u_{1}\left(e_{1}, G_{-1}\right)$ is a strictly convex function that takes value zero at $e_{1}=0$ and at $e_{1}=\left(\alpha_{d}\right)^{2} \beta^{2} /\left(\alpha_{1}(1-\beta)\right)$. Moreover, it is strictly decreasing at $e_{1}=0$. Thus the most profitable deviation is either $e_{1}=0$ or $e_{1}=r_{l}$. From the continuity of $G_{d}$ and the arguments for $e_{1} \in\left[r_{l}, \alpha_{d}\right]$, it follows then that the payoff for $e_{1} \in\left[0, r_{l}\right]$ cannot exceed contestant 1's equilibrium payoff. Thus, the strategies in the statement constitute an equilibrium, because for each agent, given the strategies of the other players, there is no positive effort level that yields a strictly higher payoff.

Suppose now $\beta>\hat{\beta}$. Assume that contestant 1 and the disadvantaged agents employ (8) and (9), respectively. By the same arguments as before, the expected payoff of contestant 1 is $u_{1}\left(e_{1}, G_{-1}\right)=\hat{\beta}-\beta$. Under the assumption that $\beta>\hat{\beta}$, this payoff is strictly negative and contestant 1 can profitably deviate by reducing his bid to zero.

Consider now part (ii). By Lemma 2 the set of active agents is either $D$ or $N$. If it is $D$, then contestant 1 abstains and the unique equilibrium is described in Proposition 1. Consider contestant 1 and assume he bids $e_{1}=\alpha_{d}$. This yields $u_{1}\left(e_{1}, G_{-1}\right)=1-\beta-$ $\alpha_{d} / \alpha_{1}=\hat{\beta}-\beta>0$. Now suppose the set of active agents is $N$. Then by Claim 3 in the proof of Lemma 2 and the fact that each point must be a point of increase for at least two contestants (Lemma 7 in Baye et al., 1996, applies), there must exist $e \in\left(0, r_{d}\right]$ which is

\footnotetext{
${ }^{30}$ Details are available upon request.
} 
a point of increase for all three contestants. For each such $e$ the only compatible cdfs are described in (8) and (9). Lastly notice that we cannot have that $\underline{s}_{1}>r_{l}$, as this would require that contestant 1 places an atom at zero and hence implies zero payoffs. Thus, (8) and (9) describe the unique equilibrium.

Q.E.D.

\section{A.6 Proof of Proposition 3}

Notice first that $G_{1}\left(e_{1}\right)$ and $G_{d}\left(e_{d}\right)$ as defined in (11) and (12) are well defined distribution functions.

Suppose $\beta=\hat{\beta}$ and that contestant 1 and a disadvantaged agent, say contestant 2 , employ (11) and (12). The expected payoff of contestant 3 for any $e_{3}$ is

$$
u_{3}\left(e_{3}, G_{-3}\right)=\left\{\begin{array}{ll}
\frac{e_{3}}{\left((1-\beta) \gamma_{1}(0)+\beta\right) \alpha_{d}}\left((1-\beta) \gamma_{1}(0)+\beta\right)-\frac{e_{3}}{\alpha_{d}} & \text { if } e_{3} \in[0, \lambda] \\
\sqrt{\frac{e_{3}}{\alpha_{d}}}\left(\left(\frac{\alpha_{1}}{\alpha_{d}} \sqrt{\frac{e_{3}}{\alpha_{d}}}-\frac{\alpha_{1}-\alpha_{d}}{\alpha_{d}}\right)(1-\beta)+\beta\right)-\frac{e_{3}}{\alpha_{d}} & \text { if } e_{3} \in\left[\lambda, \alpha_{d}\right] . \\
1-\frac{e_{3}}{\alpha_{d}} & \text { if } e_{3} \geq \alpha_{d}
\end{array} .\right.
$$

Using that $\beta=\hat{\beta}$ and simplifying, we obtain that $u_{3}\left(e_{3}, G_{-3}\right) \leq 0$, as

$$
u_{3}\left(e_{3}, G_{-3}\right)=\left\{\begin{array}{ll}
\frac{e_{3}}{\alpha_{d}}-\frac{e_{3}}{\alpha_{d}} & \text { if } e_{3} \in[0, \lambda] \\
\sqrt{\frac{e_{3}}{\alpha_{d}}} \sqrt{\frac{e_{3}}{\alpha_{d}}}-\frac{e_{3}}{\alpha_{d}} & \text { if } e_{3} \in\left[\lambda, \alpha_{d}\right] . \\
1-\frac{e_{3}}{\alpha_{d}} & \text { if } e_{3} \geq \alpha_{d}
\end{array} .\right.
$$

Suppose now that agents 2 and 3 follow the strategies in the statement. Consider agent 1. We have that

$$
u_{1}\left(e_{1}, G_{-1}\right)= \begin{cases}\left(\frac{e_{1}}{\left((1-\beta) \gamma_{1}(0)+\beta\right) \alpha_{d}}\right)^{2}(1-\beta)-\frac{e_{1}}{\alpha_{1}} & \text { if } e_{1} \in[0, \lambda] \\ \frac{e_{1}}{\alpha_{d}}(1-\beta)-\frac{e_{1}}{\alpha_{1}} & \text { if } e_{1} \in\left[\lambda, \alpha_{d}\right] \\ 1-\beta-\frac{e_{1}}{\alpha_{1}} & \text { if } e_{1} \geq \alpha_{d}\end{cases}
$$

Again using that $\beta=\hat{\beta}$ and simplifying, we obtain

$$
u_{1}\left(e_{1}, G_{-1}\right)= \begin{cases}\left(\frac{e_{1}}{\left((1-\beta) \gamma_{1}(0)+\beta\right) \alpha_{d}}\right)^{2} \frac{\alpha_{d}}{\alpha_{1}}-\frac{e_{1}}{\alpha_{1}} & \text { if } e_{1} \in[0, \lambda] \\ \frac{e_{1}}{\alpha_{1}}-\frac{e_{1}}{\alpha_{1}} & \text { if } e_{1} \in\left[\lambda, \alpha_{d}\right] \\ \frac{\alpha_{d}}{\alpha_{1}}-\frac{e_{1}}{\alpha_{1}} & \text { if } e_{1} \geq \alpha_{d}\end{cases}
$$

To conclude that $u_{1}\left(e_{1}, G_{-1}\right) \leq 0$ it remains to show that for $e_{1} \in[0, \lambda]$ it holds that

$$
\left(\frac{e_{1}}{\left((1-\beta) \gamma_{1}(0)+\beta\right) \alpha_{d}}\right)^{2} \frac{\alpha_{d}}{\alpha_{1}}-\frac{e_{1}}{\alpha_{1}} \leq 0 \Leftrightarrow e_{1} \leq\left((1-\beta) \gamma_{1}(0)+\beta\right)^{2} \alpha_{d}=\lambda .
$$

This, of course, is true in the first branch of (27).

Thus, the strategies in the statement constitute an equilibrium, because for each agent, given the strategies of the other players, there is no positive effort level that yields a strictly higher payoff.

Q.E.D. 


\section{A.7 Proof of Proposition 5}

We start with the following claim that characterises the expected sum of effort in the continuum of equilibria for $\beta=\hat{\beta}$.

Claim 8. The expected sum of effort in the continuum of equilibria for $\beta=\hat{\beta}$ (described in Proposition 3) is given by

$$
\alpha_{d}+\frac{\alpha_{1}-\alpha_{d}}{3}\left(1-\left((1-\hat{\beta}) \gamma_{1}(0)+\hat{\beta}\right)^{3}\right) .
$$

This expression is strictly decreasing in $\gamma_{1}(0)$ and equals $\alpha_{d}$ for $\gamma_{1}(0)=1$.

Proof: We first derive (28). The expected effort of contestant 1 is

$$
\begin{aligned}
E\left(e_{1}\right) & =\int_{\lambda}^{\alpha_{d}} g_{1}\left(e_{1}\right) e_{1} d e_{1}=\int_{\lambda}^{\alpha_{d}} \frac{\alpha_{1}}{\alpha_{d}} \frac{\sqrt{e_{1}}}{2 \sqrt{\alpha_{d}}} d e_{1}=\left.\frac{\alpha_{1}}{\alpha_{d}} \frac{\left(e_{1}\right)^{3 / 2}}{3 \sqrt{\alpha_{d}}}\right|_{\lambda} ^{\alpha_{d}} \\
& =\frac{\alpha_{1}}{3}\left(1-\left((1-\hat{\beta}) \gamma_{1}(0)+\hat{\beta}\right)^{3}\right) .
\end{aligned}
$$

The expected effort of a disadvantaged contestant is

$$
\begin{aligned}
E\left(e_{d}\right) & =\int_{0}^{\lambda} g_{d}\left(e_{d}\right) e_{d} d e_{d}+\int_{\lambda}^{\alpha_{d}} g_{d}\left(e_{d}\right) e_{d} d e_{d} \\
& =\int_{0}^{\lambda} \frac{e_{d}}{\alpha_{d}\left((1-\hat{\beta}) \gamma_{1}(0)+\hat{\beta}\right)} d e_{d}+\int_{\lambda}^{\alpha_{d}} \frac{\sqrt{e_{d}}}{2 \sqrt{\alpha_{d}}} d e_{d} \\
& =\left.\frac{\left(e_{d}\right)^{2}}{2 \alpha_{d}\left((1-\hat{\beta}) \gamma_{1}(0)+\hat{\beta}\right)}\right|_{0} ^{\lambda}+\left.\frac{\left(e_{d}\right)^{3 / 2}}{3 \sqrt{\alpha_{d}}}\right|_{\lambda} ^{\alpha_{d}} \\
& =\frac{\alpha_{d}}{6}\left((1-\hat{\beta}) \gamma_{1}(0)+\hat{\beta}\right)^{3}+\frac{\alpha_{d}}{3}
\end{aligned}
$$

The expected sum of effort is hence

$$
\frac{\alpha_{1}}{3}\left(1-\left((1-\hat{\beta}) \gamma_{1}(0)+\hat{\beta}\right)^{3}\right)+\frac{\alpha_{d}}{3}\left((1-\hat{\beta}) \gamma_{1}(0)+\hat{\beta}\right)^{3}+\frac{2 \alpha_{d}}{3}
$$

which is the same as 28). It is straightforward to verify that 28 equals $\alpha_{d}$ for $\gamma_{1}(0)=1$ and strictly decreases with $\gamma_{1}(0)$ under our assumption that $0<\alpha_{d}<\alpha_{1}$.

To conclude the proof notice that for $\beta<\hat{\beta}$ the unique equilibrium is described in Proposition 2. Moreover, since the associated density functions to the cdfs in (8) and (9) are continuous in $\beta$, it follows that the expected sum of effort is also continuous in $\beta$. In addition, the statement of Proposition 2 includes the case of $\beta=\hat{\beta}$ in which case the expressions in (8) and (9) reduce to the equilibrium in Proposition 3 described by (11) and 12 with $\gamma_{1}(0)=0$. This implies that for $\beta<\hat{\beta}$ large enough, the expected sum of effort in the unique equilibrium is strictly larger than $\alpha_{d}$, the expected sum of effort when the advantaged agent is excluded.

Q.E.D. 


\section{A.8 Proof of Lemma 3}

Consider any $\beta>0$ and any equilibrium described in Propositions 17 . Notice that if the additional agents bid zero with probability one, then it is an equilibrium in the modified game that the original agents bid as in the original equilibrium. Hence, it remains to show that if the original agents bid as specified in the original equilibrium and all but one additional agent bid zero with probability one, then there is no positive effort level that yields the remaining additional agent, say $i$, a strictly positive payoff.

Suppose first that there exists an agent, say $j$, of the same type (advantaged or disadvantaged) with higher ability $\alpha_{i} \leq \alpha_{j}$ in the original game. If this agent is advantaged assume that the equilibrium payoff in the original equilibrium is zero. Notice that if agent $j$ is disadvantaged, from Propositions 13 we can conclude that agent $j$ 's equilibrium payoff in the original equilibrium is zero, too. Thus, given the equilibrium strategies $G_{k}$ and $G_{h}$ of the other original agents, with agent $k$ disadvantaged, player $j$ 's expected payoff from any $\hat{e}$ is

$$
u_{j}\left(\hat{e}, G_{-j}\right)=G_{k}(\hat{e})\left(G_{h}(\hat{e})(1-\beta)+z_{j} \beta\right)-\frac{\hat{e}}{\alpha_{j}} \leq 0,
$$

where $z_{j} \in\{0,1\}$ takes value 1 if $j \in D$, and value 0 otherwise. Player $i$ 's expected payoff from the same effort level $\hat{e}$ is then

$$
u_{i}\left(\hat{e}, G_{-i}\right)=G_{j}(\hat{e}) G_{k}(\hat{e})\left(G_{h}\left(e_{j}\right)(1-\beta)+z_{i} \beta\right)-\frac{\hat{e}}{\alpha_{i}} \leq 0,
$$

as $G_{j}(\hat{e}) \leq 1$ and $\alpha_{i} \leq \alpha_{j}$.

Suppose lastly that the additional agent $i$ is advantaged and that there exists a disadvantaged agent $j$ with higher ability $\alpha_{i} \leq \alpha_{j}$ in the original game. Agent $j$ 's expected payoff from any $\hat{e}$ is

$$
u_{j}\left(\hat{e}, G_{-j}\right)=G_{k}(\hat{e})\left(G_{h}(\hat{e})(1-\beta)+\beta\right)-\frac{\hat{e}}{\alpha_{j}} \leq 0,
$$

while player $i$ 's expected payoff from the same effort level $\hat{e}$ is then

$$
u_{i}\left(\hat{e}, G_{-i}\right)=G_{j}(\hat{e}) G_{k}(\hat{e}) G_{h}\left(e_{j}\right)(1-\beta)-\frac{\hat{e}}{\alpha_{i}} \leq 0,
$$

as $z_{i}=0, G_{j}(\hat{e}) \leq 1$ and $\alpha_{i} \leq \alpha_{j}$.

Q.E.D.

\section{References}

Alcalde, J. and M. Dahm. "Tullock and Hirshleifer: A Meeting of the Minds." Review of Economic Design, 11(2), 2007, 193-208. 
Alcalde, J. and M. Dahm. "Rent Seeking and Rent Dissipation: A Neutrality Result." Journal of Public Economics, 94(1-2), 2010, 1-7.

Arbatskaya, M. "The Exclusion Principle for Symmetric Multi-Prize All-Pay Auctions with Endogenous Valuations." Economics Letters, 80, 2003, 73-80.

Baye M.R., D. Kovenock, and C.G. de Vries. "Rigging the Lobbying Process: An Application of the All-Pay Auction." American Economic Review, 83(1), 1993, 289-294.

Baye M.R., D. Kovenock, and C.G. de Vries. "The All-Pay Auction with Complete Information." Economic Theory, 8, 1996, 291-305.

Barut, Y. and D. Kovenock. "The Symmetric Multiple Prize All-Pay Auction with Complete Information." European Journal of Political Economy, 14, 1998, 627-644.

Bertoletti, P. "A Note on the Exclusion Principle." Journal of Mathematical Economics, 44, 2008, 1215-1218.

Beviá, C. and L.C. Corchón. "Centralized vs Decentralized Contests." Economics Letters, 137, 2015, 32-35.

Clark, D. and C. Riis. "Competition Over More than One Prize." American Economic Review, 88, 1998, 276-289.

Cohen, C. and A. Sela. "Allocation of Prizes in Asymmetric All-Pay Auctions." European Journal of Political Economy, 24, 2008, 123-132.

Dahm, M. and P. Esteve-González. "Affirmative Action Through Extra Prizes." Journal of Economic Behavior and Organization, 153, 2018, 123-142.

Fang, H. "Lottery Versus All-Pay Auction Models of Lobbying." Public Choice, 112(3-4), 2002, 351-371.

Franke, J., C. Kanzow, W. Leininger, and A. Schwartz. "Lottery Versus All-Pay Auction Contests: A Revenue Dominance Theorem." Games and Economic Behavior, 83, 2014, $116-126$.

Franke, J., W. Leininger, and C. Wasser. "Optimal Favoritism in All-Pay Auctions and Lottery Contests." European Economic Review, 104, 2014, 22-37.

Fu, Q. "A Theory of Affirmative Action in College Admissions." Economic Inquiry, 44(3), 2006, 420-428.

Fu, Q. and J. Lu. "Competitive Effect of Cross-Shareholdings in All-Pay Auctions with Complete Information." Internation Journal of Industrial Organization, 31, 2013, 267277. 
Glazer, A. and R. Hassin. "Optimal Contests." Economic Inquiry, 26(1), 1988, 133-143.

Hillman, A.L. and D. Samet. "Dissipation of Contestable Rents by a Small Number of Contenders." Public Choice, 54, 1987, 63-82.

Hillman, A.L. and J.G. Riley. "Politically Contestable Rents and Transfers." Economics and Politics, 1, 1989, 17-39.

Konrad, K.A. "Silent Interest and All-Pay Auctions." International Journal of Industrial Organization, 24, 2006, 701-713.

Li, S. and J. Yu. "Contests with Endogenous Discrimination." Economics Letters, 117, 2012, 834-836.

Liu, X. and J. Lu. "Optimal Prize-Rationing Strategy in All-Pay Contests with Incomplete Information." Internation Journal of Industrial Organization, 50, 2017, 57-90.

Matros, A. and D. Rietzke. "Contests on Networks." Working Paper 2017/006, Lancaster University, 2017.

Menicucci, D. "Banning Bidders from All-Pay Auctions." Economic Theory, 29, 2006, 89-94.

Moldovanu, B. and A. Sela. "The Optimal Allocation of Prizes in Contests." American Economic Review, 91, 2001, 542-558.

Pastine, I. and T. Pastine. "Student Incentives and Preferential Treatment in College Admisssions." Economics of Education Review, 31, 2012, 123-130.

Serena M. "Quality Contests." European Journal of Political Economy, 46, 2017, 15-25.

Siegel, R. "All-Pay Contests." Econometrica, 77(1), 2009, 71-92.

Siegel, R. "Asymmetric Contests with Conditional Investments." American Economic Review, 100(5), 2010, 2230-60.

Siegel, R. "Asymmetric Contests with Head Starts and Nonmonotonic Costs." American Economic Journal: Microeconomics, 6(3), 2014, 59-105.

Sisak, D. "Multiple-Prize Contests - The Optimal Allocation of Prizes." Journal of Economic Surveys, 23(1), 2009, 82-114.

Szymanski S. and Valletti TM. "Incentive Effects of Second Prizes." European Journal of Political Economy, 21, 2005, 467-481.

Xiao, J. "Asymmetric All-Pay Contests with Heterogeneous Prizes." Journal of Economic Theory, 163, 2016, 178-221. 